\title{
ADITIVO LIBERADOR DE TUBERÍAS DE PERFORACIÓN A BASE DE DESTILADO DE COQUE DE PETRÓLEO
}

\section{DRILLING PIPE RELEASE ADDITIVE BASED ON PETROLEUM COKE DISTILLATE}

\author{
Rubén Darío Vega Mejía ${ }^{1}$ \\ Oskarina Del Valle Rodríguez González ${ }^{2}$ \\ Adnel Miguel Rodríguez Figuera ${ }^{3}$
}

\begin{abstract}
RESUMEN
El objetivo fundamental de esta investigación fue desarrollar un aditivo liberador de tubería a base de destilados de coque de petróleo. Para ello, se empleó un extractor soxhlet usando xileno como solvente orgánico. Posteriormente, se estableció la formulación de las diferentes píldoras liberadoras de tubería (con aditivo comercial y con destilado de coque), estudiándose el efecto de estas sobre las propiedades de los diferentes revoques generados a partir de un fluido de perforación $100 \%$ aceite mineral mediante pruebas de calidad a nivel de laboratorio, tales como: coeficiente de lubricidad, pérdida de peso y disminución del espesor de revoque. Se aplicó una Anova para un diseño experimental de bloques aleatorios simples, conformado por cuatro bloques (píldoras), tres tratamientos (pruebas de calidad) y cuatro repeticiones; y un Test de Duncan $(\alpha=0,05)$, demostrándose que los tratamientos con destilado de coque presentaron mejor comportamiento con respecto a la píldora comercial. Se concluyó, que la diferencia entre las píldoras se debe al mecanismo de acción de cada una.
\end{abstract}

Palabras clave: coeficiente de fricción, destilado de coque, pega de tubería, reducción de espesor, reducción de peso.

\begin{abstract}
The fundamental objective of this research was to develop a pipe-releasing additive based on petroleum coke distillates. For this, a soxhlet extractor was used using xylene as an organic solvent. Subsequently, the formulation of the different pipe release pills (with commercial additive and coke distillate) was established, studying the effect of these on the properties of the different plasters generated from a 100\% mineral oil drilling fluid through tests quality at laboratory level, such as: lubricity coefficient, weight loss and reduction of the thickness of the plaster. An Anova was applied for an experimental design of simple randomized blocks, consisting of four blocks (pills), three treatments (quality tests) and four repetitions; and a Duncan Test $(\alpha=0.05)$, showing that the treatments with coke distillate presented better
\end{abstract}

\footnotetext{
${ }^{1}$ Ingeniero Mecánico, profesor Asociado de la Universidad de Oriente, docente investigador de la misma universidad. MSc en Mantenimiento Industrial y Gerencia Empresarial. rubendvegam@gmail.com, (D) 0000-0002-6331-3851 2 Universidad de Oriente, Núcleo de Monagas. Escuela de Ingeniería y Ciencias Aplicadas (EICA), Departamento de Ingeniería de Petróleo, Campus Los Guaritos. Avenida Universidad, Maturín Monagas-Venezuela. Karirodriguez10.or@gmail.com, (D)0000-0001-6195-5917

3 Ingeniero de Petróleo de la Universidad de Oriente. adnelr123@gmail.com, (D)0000-0003-2092-6882
} 
performance compared to the commercial pill. It was concluded that the differences between the pills is due to the mechanism of action of each one.

Keywords: coefficient of friction, coke distillate, pipe stuck, thickness reduction, weight reduction.

\section{INTRODUCCIÓN}

El fluido de perforación es una parte inseparable de las operaciones modernas de perforación, siendo esencial para cumplir varias tareas funcionales y garantizar una operación segura, sin problemas, económica y rentable (Amanullah, 2016). Esto lo logra mediante la realización de múltiples funciones como: enfriar la broca, controlar las presiones, lubricar la tubería de perforación, asegurar la formación del revoque y evitar daños a la formación. Sí el fluido falla en alguna de sus funciones se podrían presentar graves problemas como: pérdida de circulación, daños a la formación, pega de tubería, erosión de la formación y altos valores de torque y arrastre que reducen significativamente la eficiencia de la perforación (Hoelscher et al., 2012). Además, conlleva a la presencia de tiempos no productivos (TNP), el cual es uno de los principales desafíos operativos y fiscales del proceso de perforación, responsable de miles de millones de dólares en costos ocultos (Hilfiger et al., 2017).

La mayor parte de los TNP a menudo se atribuyen a eventos de pega de tubería, porque este es uno de los problemas de perforación más comunes durante la perforación. Y es que reportes técnicos señalan que, el $36 \%$ de los problemas de perforación registrados en todo el mundo se debieron a tuberías atascadas (Reid et al,. 2000). Este inconveniente produce que la sarta de perforación no pueda ni rotar ni desplazarse a lo largo del pozo. De acuerdo a la complejidad de la pega de tubería puede a conllevar a aumentos de los costos en las operaciones, resultados negativos y hasta una posible desviación del hoyo o abandono del pozo.

Dos fenómenos diferentes son las causas de la pega de tubería, que se reflejan en sus términos comunes: tubería atascada mecánicamente y tubería atascada diferencialmente. La adherencia mecánica de la tubería se refiere al proceso por el cual algo bloquea físicamente la extracción de la tubería. La incapacidad de hacer circular el fluido del pozo desde el fondo es otro problema importante, y puede provocar que el asentamiento de los recortes provoque que la tubería se atasque (Karimi et al., 2011). Una de las razones de la pega de tubería ocurre por la mala o pobre lubricación sobre la misma. La función principal de un lubricante es reducir el coeficiente de fricción entre superficies en movimiento y ofrecer protección contra el desgaste de las herramientas.

Existen agentes lubricantes sólidos y líquidos (liberadores de tuberías), la mayoría de los lubricantes sólidos están basados en micro o nano esferas que actúan como rodamientos de bolas para crear una superficie resbaladiza o deslizante en la interfaz formación- tubería. Los lubricantes líquidos crean una película delgada en dicha interfaz para neutralizar el efecto de la rugosidad de la superficie y permitir un deslizamiento fácil de la superficie en movimiento con respecto a la superficie estática (Amanullah, 2016). Los liberadores de tuberías consisten de detergentes, jabones, aceites, surfactantes y otros materiales químicos. Las prácticas de campo muestran que la mayoría de los aditivos liberadores de tubería (píldoras) adicionados a los fluidos de perforación tienen problemas complejos, como lubricación insatisfactoria y poca resistencia al desgaste (Zhang et al., 2013). Los fluidos base aceite naturales y sintéticos generalmente producen un coeficiente de fricción menor a aquellos producidos por fluidos base agua (Growcock et al., 1999).

Actualmente, estudios como los de Amanullah, (2016), Hilfiger et al., (2017), Yunfeng et al., (2018) y Foxenberg et al., (2008) entre otros, se han llevado a cabo para desarrollar aditivos liberadores de 
tubería líquidos. Los mismos tienen como principal propósito reducir el coeficiente de lubricación basado en reutilización de aceites vegetales, aplicación de emulsiones o nuevos liberadores de tubería. Respecto al coque de petróleo, Venezuela cuenta con las más grandes reservas de crudo pesado y extrapesado del mundo, la mayoría ubicadas en la Faja Petrolífera del Orinoco (Valera et al., 2013). Asimismo, existen considerables acumulaciones de coque de petróleo en el estado Anzoátegui, específicamente en el Complejo Petroquímico General de División José Antonio Anzoátegui, como consecuencia del proceso de coquización retardada, al cual es sometido el crudo de la Faja (Ministerio del Poder Popular para la Educación Universitaria, Ciencia y Tecnología [MPPEUCT], 2012).

Bajo este contexto, la presente investigación tuvo como propósito la formulación de un aditivo liberador de tubería a base de destilado de coque de petróleo, con el fin de darle uso al coque de petróleo almacenado en Venezuela. Todo ello conforme a la línea de investigación denominada "Desarrollo de aditivos no convencionales" desplegada en el laboratorio de fluidos de perforación del Departamento de Ingeniería de Petróleo de la Universidad de Oriente, Núcleo de Monagas, Venezuela.

\section{METODOLOGÍA}

Para la realización de la investigación se empleó una muestra de coque de petróleo del complejo mejorador PetroCedeño de Petróleo de Venezuela S.A [PDVSA], ubicado en el estado AnzoáteguiVenezuela. La elaboración del aditivo liberador de tubería, partió de la extracción del aceite contenido en el coque a través del método del Soxhlet, Método 3540C (Environmental Protection Agency [EPA], 1996), utilizando como solvente orgánico xileno. El producto obtenido de la extracción se sometió a un proceso de destilación, aplicando la norma ASTM D86-19 (American Society for Testing and Materials [ASTM], 2019).

\section{Caracterización del destilado de coque de petróleo}

Obtenido el destilado de coque de petróleo este se sometió a una Espectroscopia de Infrarrojo de Transformada de Fourier, utilizando un equipo Perkin Elmer FTIR 1600. Posteriormente, mediante procedimientos estandarizados se conocieron las propiedades reflejadas en catálogos de liberadores de tuberías comerciales, como lo son: gravedad específica (Norma ASTM D369, (ASTM, 2002)), viscosidad cinemática y dinámica (Norma ASTM D 2196, (ASTM, 2010)), punto de inflamación (Norma ASTM D-92, (ASTM, 2014)) y pH (norma ASTM D3838, (ASTM, 1980)).

\section{Estudio del destilado como aditivo liberador de tuberías}

Inicialmente se formularon cuatro (4) píldoras equivalentes para ser empleadas como aditivos liberadores de tuberías (Tabla 1).

Tabla 1

Formulación de las píldoras liberadoras de tubería producto

\begin{tabular}{|c|c|c|c|c|}
\hline Denominación & Píldora $1\left(P_{1}\right)$ & Píldora $2\left(\mathbf{P}_{2}\right)$ & Píldora $3\left(P_{3}\right)$ & Píldora $4\left(P_{4}\right)$ \\
\hline Liberador Comercial (PEG-350) (mL) & 200 & 100 & - & - \\
\hline Aceite mineral Vassa LP40 (mL) & - & 100 & 100 & - \\
\hline \multirow[t]{2}{*}{ Liberador Propuesto (mL) } & - & - & 100 & 200 \\
\hline & $100 \%$ PEG-350 & $\begin{array}{c}50 \% \text { PEG-350 / } \\
50 \% \text { Vassa LP40 }\end{array}$ & $\begin{array}{c}\text { 50\% Aceite de coque de } \\
\text { petróleo / } 50 \% \text { Vassa } \\
\text { LP40 }\end{array}$ & $\begin{array}{l}\text { 100\% Aceite de } \\
\text { coque de } \\
\text { petróleo }\end{array}$ \\
\hline
\end{tabular}

Una primera píldora de $100 \%$ liberador comercial de la empresa Schlumberger y una segunda píldora $50 \%$ liberador comercial - 50\% aceite Vassa, las cuales sirvieron como píldoras de control; la tercera píldora formulada con una relación píldora $50 \%$ liberador propuesto $-50 \%$ aceite Vassa y una última $100 \%$ liberador propuesto. Cada una de ellas se mezclaron de manera independiente 
durante 5 min siguiendo la metodología planteada por Esvenca, (2007).

Bajo la formulación de un fluido de perforación 100\% aceite mineral con una densidad de 11 lpg (Esvenca, 2007) (Tabla 2) se prepararon tres (3) barriles para cada píldora en estudio (12 en total) y tres (3) adicionales considerados como control (sin píldora). Aplicando la norma API RP 13B-2, los mismos fueron envejecidos con la finalidad de simular las condiciones de yacimiento, este proceso consistió en someter al fluido a una temperatura de $300^{\circ} \mathrm{F}$, una presión de $100 \mathrm{lpcm}$ por un período de 16 horas (American Petroleum Institute [API], 2005).

Tabla 2

Formulación del fluido $100 \%$ aceite mineral

\begin{tabular}{cc}
\hline Producto & Concentración \\
\hline Aceite Mineral $(\mathrm{mL})$ & 224,59 \\
Cal hidratada $(\mathrm{g})$ & 8 \\
Humectante $(\mathrm{mL})$ & 1 \\
Activador polar $(\mathrm{mL})$ & 10,50 \\
Arcilla organofílica $(\mathrm{g})$ & 10,00 \\
Lignito organofílico $(\mathrm{g})$ & 8 \\
Emulsificante $(\mathrm{mL})$ & 1 \\
Barita $(\mathrm{g})$ & 199,09 \\
\hline
\end{tabular}

Tomado de: Esvenca, (2007)

Basado en la metodología de Amanullah, (2016) a los fluidos contentivos de las píldoras formuladas se les determinó el coeficiente de lubricación haciendo uso de un medidor de lubricidad OFITE, el cual permitió la aplicación de las siguientes ecuaciones:

$$
\begin{gathered}
\text { Factor de corrección (FC) }=\frac{\mathrm{T}_{\text {srdw }}}{\mathrm{T}_{\mathrm{ardw}}} \\
\text { Coeficiente de fricción }(\mathbf{C O F})=\frac{\mathrm{T}_{\mathrm{rm}} * \mathrm{FC}}{100 \mathrm{lb}} \\
\% \text { Reducción COF }(\mathbf{C O F R})=\frac{\mathrm{COF}_{\mathrm{fsp}}-\mathrm{COF}_{\mathrm{fcp}}}{\mathrm{COF}_{\mathrm{fsp}}} * 100
\end{gathered}
$$

Donde:

$T_{\text {srdw }}=$ Lectura de torque estándar del fluido de calibración

$T_{\text {ardw }}=$ Lectura de torque real del fluido de calibración

$\mathrm{T}_{\mathrm{bm}}=$ Lectura de torque del fluido en estudio (sin píldora)

$\mathrm{COF}_{\mathrm{fsp}}=$ Coeficiente de fricción de un fluido sin píldora

$\mathrm{COF}_{\mathrm{fcp}}=$ Coeficiente de fricción de un fluido con píldora

A partir del método de filtrado de alta presión/alta temperatura (HT/HP) se obtuvieron los revoques de los fluidos (API, 2005), a ellos antes y después de someterlos a los efectos de las diferentes píldoras propuestas, se le observó su estado inicial y el resquebrajamiento por efecto de la píldora, se les determinó el espesor de revoque (API, 2005) y el peso del revoque. Se empleó la metodología de Wagle et al., (2015) para establecer el efecto del aditivo sobre el peso del revoque obtenido (Ecuación 4) y una adaptación de esta permitió verificar el rendimiento de la píldora para el espesor de revoque.

Donde:

$$
\% \text { reducción de peso }=\left|\frac{\mathrm{W}_{\mathrm{f}}-\mathrm{W}_{\mathrm{a}}}{\mathrm{W}_{\mathrm{f}}}\right| * 100
$$

$\mathrm{Wf}=$ Peso o espesor del revoque después de la aplicación de la píldora

Wa $=$ peso o espesor del revoque antes de la aplicación de la píldora 


\section{Análisis estadístico}

Para conocer las diferencias estadísticas significativas o no del comportamiento de los revoques sometidos a los efectos de las píldoras con aditivo comercial y destilado de coque, Se aplicó una Anova para un diseño experimental de bloques aleatorios simples, conformado por cuatro bloques (píldoras), tres tratamientos (pruebas de calidad) y cuatro repeticiones; y un Test de Duncan $(\alpha=0,05)$. Para ello se utilizó el programa estadístico InfoStat ${ }^{\circledR}$.

\section{RESULTADOS}

Una vez sometido el coque de petróleo al método soxhlet y al proceso de destilado, se obtuvo un líquido de color negro a simple vista (Figura 2), también se observó que al dejarse reposar, decantaban alguno finos (sólidos) de color negro. Ello hizo pensar que en el mismo se extrajeron ciertos componentes pesados o hubo restos de coque en él.

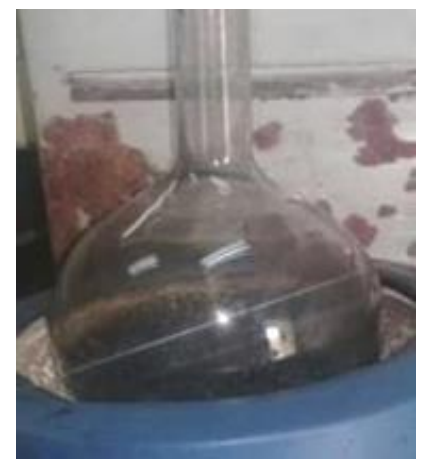

Figura 1 Destilado de coque obtenido.

En la espectroscopia IR (Figura 2), la zona entre $1000 \mathrm{~cm}^{-1}$ y $1300 \mathrm{~cm}^{-1}$ no es una zona lo suficientemente resuelta, ya que no se trata de una sustancia orgánica separada y pura, sino de una mezcla relativamente compleja.

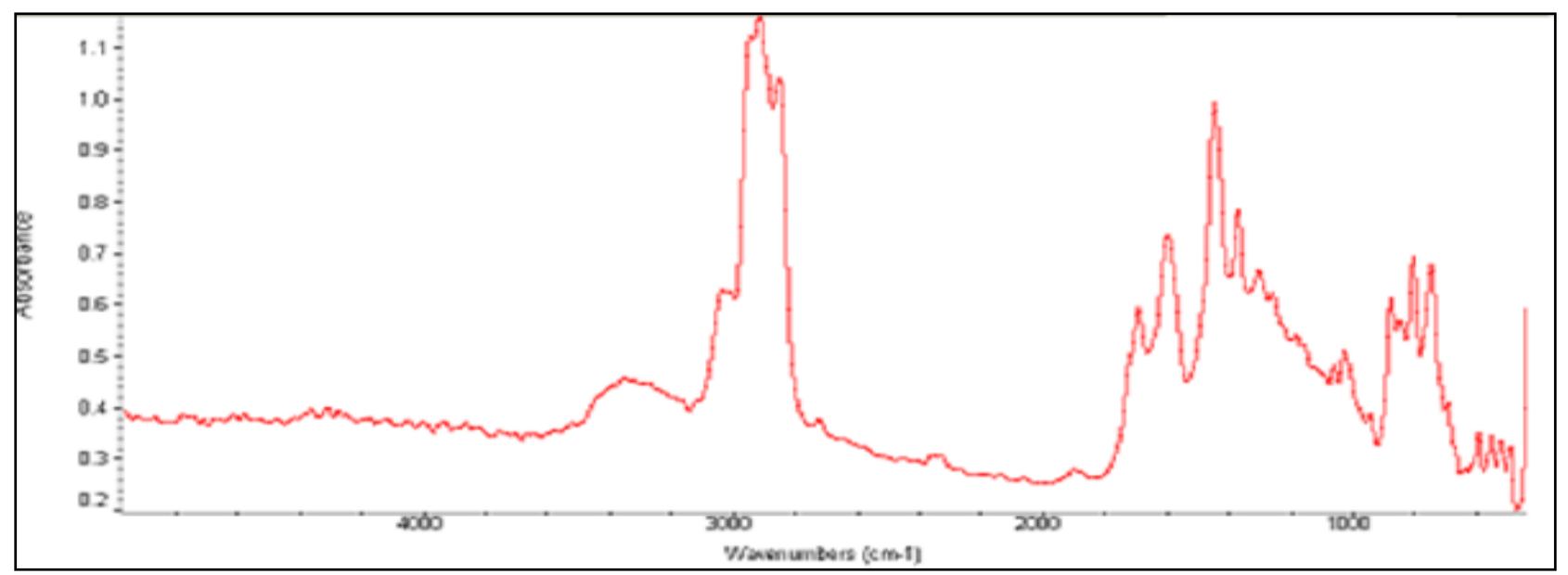

Figura 2 En la espectroscopía IR del destilado de coque

Sin embargo, se observa alrededor de $1200 \mathrm{~cm}^{-1}$ vibraciones de C-O-C, entre $1300 \mathrm{~cm}^{-1}$ y $1400 \mathrm{~cm}^{-1}$ marca de manera definida la presencia de cicloalcanos. En el rango de $1500 \mathrm{~cm}^{-1}$ y $1800 \mathrm{~cm}^{-1}$ las vibraciones están asignada a la tensión del enlace $C=C$ del anillo aromático. Entre $2800 \mathrm{~cm}^{-1}$ y 2900 $\mathrm{cm}^{-1}$ aparece un alargamiento carbón hidrógeno alifático. Además, se percibe un hombro asociado a hidrocarburos insaturados (pequeñas cantidades de aromáticos) en el rango de $2900 \mathrm{~cm}^{-1}$ y 3100 $\mathrm{cm}^{-1}$ como posiblemente residuos de xileno. Finalmente se observa una barriga entre $3100 \mathrm{~cm}^{-1} \mathrm{y}$ $3500 \mathrm{~cm}^{-1}$ producto de la existencia de alargamiento $=\mathrm{CH}$ - típicas de los compuestos aromáticos con presencia de azufre. 
El destilado obtenido reflejó de manera cualitativa una apariencia y textura igual a un aceite, del mismo modo como se observa en la tabla 3, a una temperatura de 32,2 $9 \mathrm{C}$ mostró una densidad $(\mathrm{g} / \mathrm{mL})$ y una gravedad específica de 0,8772 . Por la utilización del xileno como solvente, la densidad del destilado está relacionada con las propiedades del producto utilizado para la extracción del mismo, la densidad del xileno es aproximadamente de $0,865 \mathrm{~g} / \mathrm{mL}$. Asimismo, la gravedad específica es menor que la del agua a esa temperatura $(0,995 \mathrm{~g} / \mathrm{mL})$ indicando que el fluido estudiado posee un peso menor respecto a esta. A la misma temperatura señalada, la viscosidad dinámica y cinemática fueron de 87,15 cSt y 99,35 cP lo que supone que el destilado presente cierta dificultad para desplazarse al ser aplicado un esfuerzo cortante. Asimismo, estas propiedades son una relación entre la distancia intermolecular y la fuerza del enlace que une las mismas.

Su punto de inflamación fue de 34 o C que al ser inferior a los $100 \stackrel{\circ}{ } \mathrm{C}$ lo hace considerarlo como inflamable. Finalmente, su pH fue de 5,17 que lo cataloga como ácido de acuerdo a la escala de acidez y basicidad.

Tabla 3

Propiedades físicas y químicas del destilado de coque de petróleo y aditivo comercial.

\begin{tabular}{|c|c|}
\hline Propiedad & Resultado \\
\hline Densidad a $31,2^{\circ} \mathrm{C}(\mathrm{g} / \mathrm{mL})$ & 0,8772 \\
\hline Gravedad específica a $31,2{ }^{\circ} \mathrm{C}$ & 0,8772 \\
\hline Viscosidad dinámica a 31,2 으 (cSt) & 87,15 \\
\hline Viscosidad cinemática a 31,2 @ C (cP) & 99,35 \\
\hline Punto de inflamación (oㅡ) & 34 \\
\hline $\mathrm{pH}$ & 5,17 \\
\hline
\end{tabular}

A partir de la ecuación 3, se obtuvo el coeficiente de fricción de las cuatro píldoras, las cuales reflejaron una disminución con respecto al valor mostrado por fluido de control $(0,167)$. Los resultados se vieron afectados por el tipo de píldora empleada; en la tabla 4, se observa que el menor coeficiente de fricción lo obtuvieron las píldoras con aditivo comercial $\left(P_{1}\right.$ y $\left.P_{2}\right)$ que lo redujeron a 0,069 y reflejaron un porcentaje de reducción de 59,0\%. Los dos aditivos con destilado de coque mostraron una reducción de 0,094 y 0,092 para $\mathrm{P}_{3}$ y $\mathrm{P}_{4}$ respectivamente, a su vez exhibieron porcentajes de reducción de $43,8 \%\left(P_{3}\right)$ y $44,8 \%\left(P_{4}\right)$. Ambas píldoras evidenciaron que el adicionarle aceite Vassa no influyó en la mejora de los resultados.

Tabla 4

Valores promedios del coeficiente de fricción (COF) y \% de reducción de COF para las píldoras en estudio.

\begin{tabular}{ccc}
\hline & Coeficiente de fricción (COF) & \% Reducción de COF \\
\hline Fluido control & 0,167 & --- \\
P1 & 0,069 & 59,0 \\
P2 & 0,069 & 59,0 \\
P3 & 0,094 & 43,8 \\
P4 & 0,092 & 44,8 \\
\hline
\end{tabular}

Los revoques obtenidos mostraron buena apariencia, brillantes y gruesos como para ocasionar pega de tubería, los mismos reflejaron valores que oscilaron entre los $5,5 \mathrm{~mm}$ y 7,5 $\mathrm{mm}$ de espesor (Figura 3).

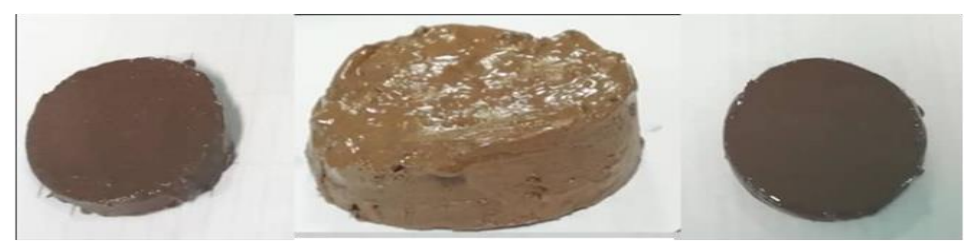

Figura 3 Ejemplos de revoques obtenidos con las píldoras empleadas 
Estos al ser sometidos a las diferentes píldoras mostraron el daño esperado (Figura 4), por lo que visualmente se puede decir que todas las píldoras cumplen con la función para la cual fueron elaboradas, eliminar la pega de tubería. Sin embargo, se observa que el volumen de los revoques en las figuras $4(\mathrm{a})$ y $4(\mathrm{~b})$ sometidas a la píldora comercial, son mayores a los volúmenes de los revoques 4 (c) y 4 (d) vinculados a las píldoras con coque de petróleo. Pero, los revoques expuestos al liberador comercial al secarse presentaron un comportamiento frágil que hizo que se partieran al manipularlos. No obstante, fue posible apreciar un mayor daño con la píldora de coque de petróleo $\mathrm{P}_{4}$ (Figura 4 (d)), pero, esto es cualitativo por lo que debe ser corroborado con el avance del presente estudio.

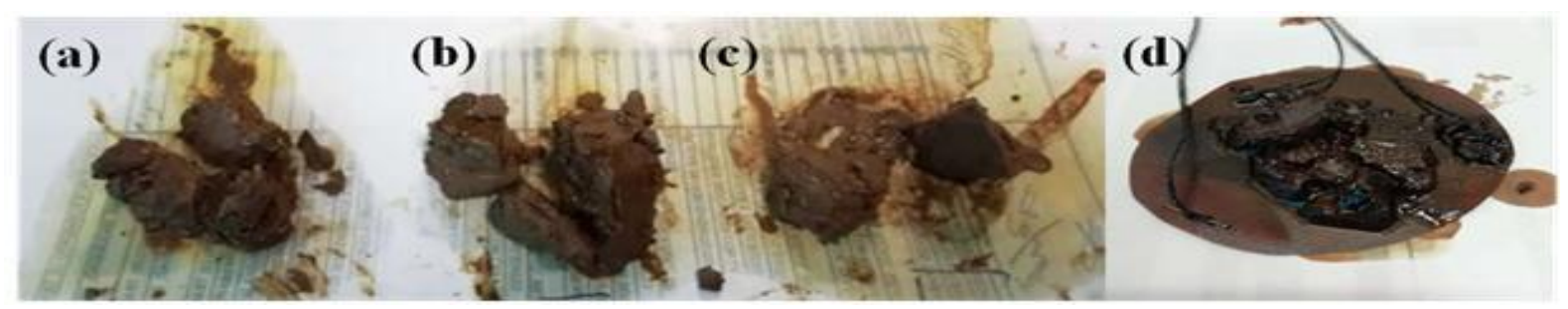

Figura 4 Ejemplos de destrucción del revoque con las píldoras empleadas. $P_{1}(a), P_{2}(b), P_{3}(c)$ y $P_{4}(d)$

Los valores promedios iniciales del peso de los revoques fluctuaron entre los $11 \mathrm{~g}$ y los $12,8 \mathrm{~g}$ (Tabla $5)$, en ellos se observó que los menores porcentajes de reducción de espesor fueron de las píldoras con el aditivo comercial, siendo estos de 3,76\% para $\mathrm{P} 1$ y $1,36 \%$ para $\mathrm{P}_{2}$. Mientras que las dos píldoras con destilado de coque reflejaron valores promedios de $21,7 \%$ para $\mathrm{P}_{3}$ y $24,8 \%$ para $\mathrm{P}_{4}$.

\section{Tabla 5}

Valores promedios del peso del revoque antes de las píldoras (ADP) y después de píldoras (DDP) en estudio

\begin{tabular}{cccc}
\hline Píldora & $\begin{array}{c}\text { Peso inicial (g) } \\
\text { (Promedio) }\end{array}$ & $\begin{array}{c}\text { Peso final (g) } \\
\text { (Promedio) }\end{array}$ & \%Reducción de peso \\
\hline P1 & 12,75 & 12,27 & 3,76 \\
P2 & 11 & 10,85 & 1,36 \\
P3 & 12,6 & 9,87 & 21,7 \\
P4 & 12,8 & 9,63 & 24,8 \\
\hline
\end{tabular}

Referente al espesor de los revoques antes de las píldoras, estos mostraron valores promedios entre $5,48 \mathrm{~mm}$ y $5,52 \mathrm{~mm}$ (Tabla 6). Al igual que el peso la menor reducción de espesor promedio se atribuyen al aditivo comercial siendo los porcentajes de reducción de 1,8\% $\left(P_{1}\right)$ y $9,1 \%\left(P_{2}\right)$. Las dos píldoras con destilado de coque presentaron un mejor comportamiento porque los porcentajes de reducción de espesor aumentaron a $16,4 \%$ para $\mathrm{P}_{3}$ y $27,3 \%$ para $\mathrm{P}_{4}$.

Tabla 6

Valores promedios del espesor del revoque antes de las píldoras (ADP) y después de píldoras (DDP) en estudio

\begin{tabular}{cccc}
\hline & Espesor en $\mathbf{~ m m}$ (ADP) & Espesor en $\mathbf{~ m m}$ (DDP) & \% Reducción de espesor \\
\hline P1 & 5,51 & 5,41 & 1,8 \\
P2 & 5,48 & 4,98 & 9,1 \\
P3 & 5,5 & 4,60 & 16,4 \\
P4 & 5,52 & 4,01 & 27,3 \\
\hline
\end{tabular}

El análisis estadístico Anova (Tabla 7) evidenció, que para las propiedades en estudio los coeficientes de correlación de Pearson estuvieron entre 0,78 y 0,98; igualmente los coeficientes de variación fueron menores a 30. Esto fue un indicativo de que los datos se ajustan al modelo empleado y no hay inconvenientes con su aplicación. Además, proyectó valores de $p$-valor menores a 0,05, por lo que existen diferencias estadísticas significativas entre las propiedades de las píldoras comparadas en la investigación. 
Tabla 7

Análisis de varianza (ANOVA) para las propiedades coeficiente de fricción (COF), peso y espesor de revoque

\begin{tabular}{cccc}
\hline Propiedad & $\mathbf{R}^{\mathbf{2}}$ & $\mathbf{C . V}$ & p-valor \\
\hline COF & 0,98 & 1,44 & $<0,0001$ \\
Peso & 0,85 & 25,58 & 0,0013 \\
Espesor & 0,78 & 11,33 & 0,0049 \\
\hline
\end{tabular}

La existencia de diferencias estadísticas significativas en las propiedades analizadas, conllevó a la aplicación del Test de Duncan (Figura 5) para conocer quien la ocasiona. Con respecto al coeficiente de fricción no existen diferencias estadísticas significativas entre las píldoras $P_{1}$ y $P_{2}$ (Letras iguales, A), pero estas si difieren con respecto a las píldoras con destilado de coque (Letras B), presentando esta últimas un comportamiento semejante entre ellas. El mejor comportamiento en este caso fue para las píldoras comerciales por presentar menor valor de coeficiente de fricción.

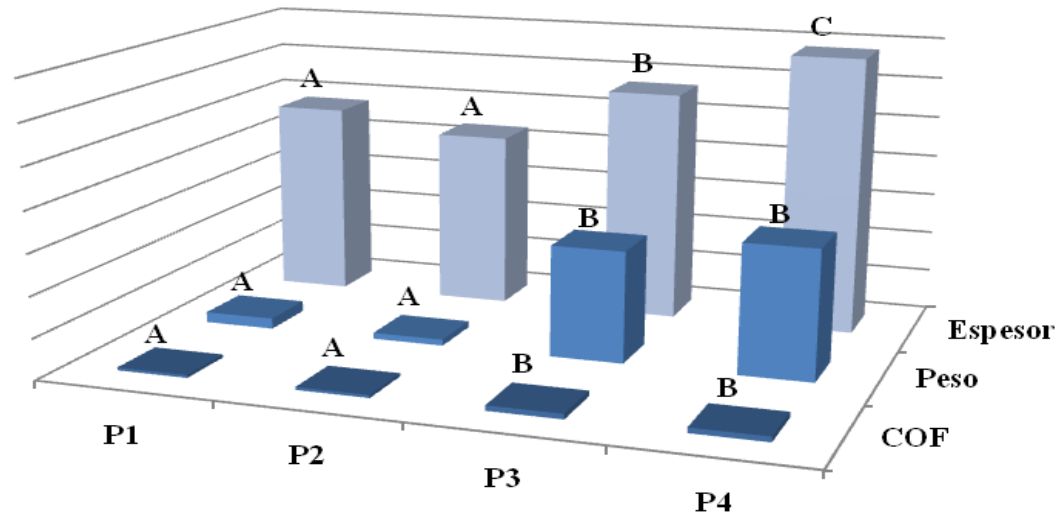

Figura 5 Test de Duncan de las propiedades COF, peso y espesor de las píldoras en estudio. (Medias con letras iguales no son estadísticamente diferentes ( $p$-valor $>0,05$ )

Asimismo, en la figura, se aprecia que, con respecto al peso de los revoques, igualmente no existió diferencias estadísticas significativas entre los tipos comunes de aditivos empleados, $\mathrm{P}_{1}$ y $\mathrm{P}_{2}$ (comercial, letra $\mathrm{A}$ ) y destilado de coque $\left(\mathrm{P}_{3}\right.$ y $\mathrm{P}_{4}$, letra $\left.\mathrm{B}\right)$, pero sí entre ellos (letras distintas). No obstante, el mejor comportamiento fue para el destilado de coque por reducir en mayor proporción el peso del revoque.

Finalmente, el espesor de revoque mostró que la píldora $\mathrm{P}_{4}$ con cien por ciento destilado de coque presentó la mayor reducción de espesor (Letra $C$ ) que ocasionó diferencias estadísticas significativas con $\mathrm{P}_{3}$ (Letra $\mathrm{B}$ ) y con $\mathrm{P}_{1}$ y $\mathrm{P}_{2}$ (letra $\mathrm{A}$ ), las cuales no difieren entre ellas.

\section{DISCUSIÓN}

Durante el proceso de extracción Soxhlet se empleó xileno como solvente orgánico y este fue sometido a calentamiento para hacerlo circular a través del coque y extraer el fluido contenido en él, por lo que se infiere que los componentes más pesados se fueron descomponiendo con la temperatura, quedando un bajo residuo de éstos presentes en el fluido obtenido, siendo esta la presunta razón por la cual el destilado presentó un color negro con presencia de sólidos. Este comportamiento concuerda y ratifica los expresado por Vega et al., (2018), quienes obtuvieron un coque más limpio despues de haberlo sometido a un proceso Soxhlet con xileno como solvente, demostrando a través de espectroscopia de infrarrojo (IR) que el xileno arrastra cierta cantidad de componentes pesados del coque de petróleo natural.

Las propiedades del aditivo comercial de Schlumberger, (2020) son: gravedad específica $(0,9 \mathrm{~g} / \mathrm{mL})$, $\mathrm{pH}(6,5)$ y punto de inflamación (38으), estas no evidencian una diferencia relevante con respecto a los valores obtenidos en el aditivo de destilado de coque. La importancia del estudio de las 
propiedades, como las viscosidades es que ellas permiten determinar la viabilidad del uso de fluido como lubricante (Mangesh et al., 2007). Los valores de viscosidad dinámica y cinemática del destilado de coque de petróleo sugirieron un comportamiento newtoniano ya que a esta temperatura demostró una viscosidad constante a los diferentes esfuerzos de cortes a los que fue sometido. Otro aspecto, importante es el señalado por Schlumberger, (2016) quien indica que una característica de los agentes empleados como liberadores de tubería es su acidez, aspecto exhibido por el aditivo propuesto.

Los resultados logrados para el coeficiente de fricción (COF) demuestran el efecto del destilado de coque sobre el fluido de control al reducir el COF de este, los mismos fueron de 0,094 y 0,092 para las muestras P3 (destilado de coque y gasoil) y P4 (destilado de coque) respectivamente, estos son menores al 0,12 mostrado por Ramirez \& Pritchard, (2020) con lubricantes sólidos. Además, son coherentes con los presentados por Amanullah, (2016) y Kingdom Drilling Services Ltd, (2008), quienes reportan valores de COF entre 0,1 y 0,2 para los fluidos base aceite o sintéticos, conllevando a expresar que estos se consideran aceptables.

El peso de los revoques estuvo entre los $11 \mathrm{~g}$ y $12,5 \mathrm{~g}$, mayores a los normalmente los alcanzados por los fluidos base aceite con distintas concentraciones de sólidos (1,7 g - 6,6 g) (Al Otaibi et al., 2008). Las reducciones de peso obtenidas con las píldoras de coque superan al obtenidos por el agente comercial, pero son menores a los señalados por Wagle et al., (2015) de 30,4\% empleando una mezcla soluble de barita y tetróxido de manganeso; aun así, son considerados como admisibles. Los espesores obtenidos estuvieron en un rango de 5,8 - 5,52 $\mathrm{mm}$, los cuales son mayores a los espesores de revoque normal ( $2 \mathrm{~mm}$ ) como los señalados por Al Otaibi et al., (2008) y Bageri et al., (2008), validando con ello que el revoque obtenido era capaz de ocasionar pega de tubería. La reducción del espesor a su vez es concordante con la pérdida de peso, es decir, a mayor pérdida de porcentaje de peso mayor pérdida de porcentaje de reducción de espesor.

Estadísticamente, quedó demostrado que el mejor desempeño fue para el destilado de coque respecto al aditivo comercial, debido a la existencia de diferencias estadísticas significativas entre los resultados de ambos. Si bien, los dos logran el objetivo deseado, su comportamiento difiere en el mecanismo de acción de cada uno, reducir el COF (comercial) o destruir el revoque (destilado de coque). Los estudios de Wagle et al., (2015) y Amanullah, (2016) solo presentaron la reducción del COF, sin evidenciar destrucción física al revoque.

\section{CONCLUSIONES}

De acuerdo a los resultados obtenidos, es posible señalar que a nivel de laboratorio es viable el uso de destilado de coque como aditivo liberador de tuberías de perforación. La diferencia en el comportamiento entre las píldoras estudiadas (comercial y destilado de coque), se debió a que el efecto de la píldora comercial es de tipo física, es decir, reduce el coeficiente de fricción y ocasiona un desgaste leve en la superficie del revoque producido, mientras que el aditivo a base de destilado de coque su efecto es químico, pues destruye el revoque, por eso la diferencia entre los porcentajes de reducción de masa y espesor. Este comportamiento se presume que se debió a la presencia de componentes pesados en el destilado y a su pH ácido. Esto lleva a sugerir el estudio del posible efecto que el destilado de coque tenga sobre las tuberías de perforación.

\section{REFERENCIAS BIBLIOGRÁFICAS}

Al Otaibi, M., Nasr-El-Din, H., \& Hill, A. (2008). Characteristics and Removal of Filter Cake Formed by FormateBased Drilling Mud. International Symposium and Exhibition on Formation Damage Control (págs. 114). Louisiana: SPE.

Amanullah, M. (2016). Coefficient of friction reducing efficiency of ARC Eco-Lube. Asia Pacific Drilling Technology Conference. Singapore. 
American Petroleum Institute [API]. (2005). Recommended Practice for Field Testing of Oil-based Drilling Fluids. API RP 13B-2. Houston.

American Society for Testing and Materials [ASTM]. (2002). Standard method for the determination of specific gravity using the Gay Lussac pycnometer. Norma ASTMD 369. West Conshohocken.

American Society for Testing and Materials [ASTM]. (2019). Standard Test Method for Distillation of Petroleum Products and Liquid Fuels at Atmospheric Pressurea. ASTM D86 - 19. West Conshohocken.

American Society for Testing and Materials [ASTM]. (2014). Standard Test Method for Flash Point by the Equilibrium Method With a Closed-Cup Apparatus, ASTM D-92. West Conshohocken.

American Society for Testing and Materials [ASTM]. (1980). Standard Test Method for $p H$ of Activated Carbon.Norma ASTM D 3838. West Conshohocken.

American Society for Testing and Materials [ASTM]. (2010). Standard Test Methods for Rheological Properties of Non-Newtonian Materials by Rotational Viscometer.Norma ASTMD 2196. West Conshohocken.

Bageri, B., Mahmoud, M., \& Al-Mutairi, S. (2008). Filter Cake Porosity and Permeability Profile Along the Horizontal Well and Their Impact on Filter Cake Removal. International Petroleum Technology Conference. Doha: SPE.

Environmental Protection Agency [EPA]. (1996). Method 3540C. Soxhlet Extraction, SW-846 Manual. Washington, DC.

Esvenca. (2007). Manual básico de fluidos de perforación. Maturín.

Foxenberg, W., Ali, S., Long, T., \& Vian., J. (2008). Field experience shows that new lubricant reduces friction and improves formation compatibility and environmental impact. International Symposium and Exhibition on Formation Damage Control held . Louisiana.

Growcock, F., Frederick, T., Reece, A., \& Green, G. (1999). Novel Lubricants for Water-Based Drilling Fluids. International Symposium on Oilfield Chemistry. Houston.

Hilfiger, M., Thaemlitz, C., \& Moellendick, E. (2017). Advanced spotting fluids for remediation of differentially stuck pipe. Middle East Oil \& Gas Show and Conference. Manama.

Hoelscher, K., De Stefano, G., Meghan, R., \& Young, S. (2012). Application of nanotechnology in drilling fluids. International Oilfield Nanotechnology Conference and Exhibition. Houston.

Karimi, M., Petrie, S., Moellendick, E., \& Holt, C. (2011). A review of casing drilling advantages to reduce lost circulation, improve wellbore stability, augment wellbore strengthening, and mitigate drillinginduced formation damage. Middle East Drilling Technology Conference \& Exhibition. Muscat.

Mangesh, G., Dalai, A., \& Bakhshi, N. (2007). Transesterification of canola oil in mixed methanol/ethanol system and use of esters as lubricity additive. Bioresource Technology (98), 2027-2033.

Ministerio del Poder Popular para la Educación Universitaria, Ciencia y Tecnología [MPPEUCT]. (2012). MPPEUCT. Recuperado el Octubre de 2020, de Ejecutivo Nacional iniciará investigaciones para el aprovechamiento del Coque de Petróleo: http://www.mppeuct.gob.ve/actualidad /noticias/ejecutivo-nacional-iniciara-investigaciones-para-el-aprovechamiento-del-coque-depetróleo

Ramirez, M., \& Pritchard, R. (2020). A Novel Solid Lubricant Uses the Principles of Tribology to Reduce theCoefficient of Friction COF in Oil- Based Muds OBM for Extended ReachDrilling Applications. Latin American and Caribbean Petroleum Engineering Conference. Bogota: SPE.

Reid, P., Meeten, G., Way, P., Clark, P., Chambers, B., Gilmour, A., y otros. (2000). Differential-sticking mechanisms and a simple wellsite test for monitoring and optimizing drilling mud properties. SPE Drill. \& Completion , 15 (2), 97-104.

Schlumberger. (2016). Fundamentos teóricos y sistemas de Dowell Schulmberger Drilling Fluids. Manual técnico de Drilling Fluids, Schlumberger. 
Schlumberger. (2020). slb.com. Recuperado el octubre de 2020, de product sheet: https://www.slb.com//media/files/mi/product-sheet/pipe-lax-w-export-ps.ashx

Valera, M., D’Elia, L., \& De Jesús, J. (2013). Una visión emergente y estratégica de utilización de coque retardado. Visión Tecnológica , 16 (1), 7-18.

Vega, R., León, M., \& Lara, P. (2018). Coque de petróleo como controlador de filtrado en fluidos de perforación base aceite. Enfoque UTE , 9 (2), 188- 197.

Wagle, V., Al-Yami, A., AlAbdullatif, Z., Bubshait, A., \& AlSafran, A. (2015). Mitigation of Stuck Pipe Challenges in HTHP Conditions Using Acid Soluble Blend of Barite and Manganese Tetroxide as Weighting Materials for Drilling Fluids. SPE North Africa Technical Conference and Exhibition , (págs. 1-13). Cairo, Egypt.

Yunfeng, L., Zhengsong, Q., \& Peng, Y. (2018). Preparation and evaluation of a high-performance drilling fluid anti- wear lubricant [J]. Drilling \& Completion Fluid , 35 (5), 1-6.

Zhang, F., Ke, M., \& Patel, A. (2013). Lubricants and drag reducers for oilfield applications-chemistry, performance, and environmental impact [C]. International Symposium on oilfield Chemistry. Houston. 